with an Esaote MyLab70XVG ultrasound device with a linear probe $(7-12 \mathrm{mHz})$ and an automated program measuring intima media thickness (IMT) by radiofrequency ("Quality intima media thickness in real-time, QIMT"), and registered the presence of atheroma plaques (per Mannheim consensus). We determined pulse wave velocity (PWV) by a validated MobilOGraph device. We considered as pathological an IMT >900 $\mu$ and a PWV $\geq 10 \mathrm{~m} / \mathrm{s}$ and the presence of plaque and/ or pathological IMT. We prospectively collected mortality and the development of new vascular events over four years and the current smoking status and exposure calculated in pack-year. Statistical analysis was performed using SPSS 17.0 software.

Results: We included 198 patients, excluding 15 because of previous CV events. The mean age was 66,5 years (SD 13,44 ) $76 \%$ were women and the mean BMI was 27,35 (SD 4,82). $31,1 \%$ were smokers, $43,2 \%$ hypertensive, $47,5 \%$ dyslipemic and $10,4 \%$ were diabetic. The mean duration of RA was 19,95 years (SD 11,88 ). $76,5 \%$ of patients were seropositive and $75,4 \%$ had erosions. The mean CRP and ESR were 9,51 mg/L (SD: 32,29) and 13,83 mm/h (SD:14,26), respectively. The mean modified SCORE was 1,81 (SD: 1,81). Regarding the vascular study, $48,1 \%$ had atheroma plaques, $32,2 \%$ a pathologic PWV [mean value of 9,13 (SD 2,12)], and $16,7 \%$ had a pathologic IMT [mean value of $748 \mu(\mathrm{DE}$ $168,73)]$.

$31.1 \%$ of the patients (57) were smokers or former smokers. The average packyear was 24.17 (SD: 21.37). No relation was found between current or previous use of tobacco and any of the outcome measures described. However, when considering cumulative exposure to tobacco, there was a trend to correlate with higher values of PWV $(p=0.07)$ and a higher plaque presence $(p=0.089)$ was detected. After 4 years of follow-up, 3 deaths were recorded among smoking patients, but a higher incidence of $\mathrm{CV}$ events was not detected in relation to cumulative exposure to tobacco $(p=0.99)$.

Conclusions: The quantification of the exposure by pack-year of cigarette smoked could give us more information about vascular damage in patients with RA. The limitation of our study is the small number of smokers in the time they were followed.

Disclosure of Interest: None declared

DOI: 10.1136/annrheumdis-2018-eular.6707

\section{AB0372 LEFT ATRIAL FUNCTION IN RHEUMATOID ARTHRITIS PATIENTS}

\section{Fawzy, S. bahoom, . Ashour, M. sheik. kaser el Aini, giza, Egypt}

Background: Rheumatoid arthritis (RA) is a common autoimmune systemic inflammatory disease affecting approximately $1 \%$ of the worldwide population. The interaction of genetic and environmental factors results in a cascade of immune reactions, which ultimately lead to the development of synovitis, joint damage, and structural bone damage. ${ }^{1}$

The importance of the left atrium in cardiovascular performance has long been acknowledged. Quantitative assessment of left atrial (LA) function is laborious, requiring invasive pressure-volume loops and thus precluding its routine clinical use. In recent years, novel postprocessing imaging methodologies have emerged, providing a complementary approach for the assessment of the left atrium. Atrial strain and strain rate obtained using either Doppler tissue imaging or two-dimensional speckle-tracking echocardiography have proved to be feasible and reproducible techniques to evaluate LA mechanics. ${ }^{2}$

Objectives:

1. To screen cardiac affection in rheumatoid arthritis patients

2. To assess subclinical echocardiography affection in RA patients

Methods: 30 healthy control, and 45 RA patient subjected to full clinical assessment, DAS 28 ESR score, full laboratory evaluation, conventional and tissue Doppler imaging (TDI) and strain (S) and strain rate (SR) analysis by two-dimensional speckle tracking of the left atrium.

Results: we found statistically significant difference in 2 Left atrial PEF, 2 Left atrial EI, 2 Left atrial TEF, TDI mitral lateral anuulus e', TDI mitral lateral annulus $\mathrm{S}$, Average SR E 1/s between patients and controls, and negative correlation between TDI lateral e, TDI lateral s, and Strain rate e and rheumatoid factor, There was negative correlation between 2LA PEF, 2LA EI, and 2LA TEF. Rheumatoid factor is correlated to strain rate $e$, and negatively correlated with left atrial passive emptying

Conclusions: RA had alteration in left LV longitudinal myocardial function, left atrial expansion volume can be a predictor of $A F$ in RA. RA patient had more left atrial stiffness. Our study concluded cardiac affection is more in seropositive RA patients.

\section{REFERENCES:}

[1] Gibofsky A. Overview of epidemiology, pathophysiology, and diagnosis of rheumatoid arthritis. Am J Manag Care 2012;18(13):S295-302.
[2] Maria J Vieira, Rogério Teixeira, LinoGonçalves, et al. Left Atrial Mechanics: Echocardiographic Assessment and Clinical Implications 2014;27 (5):463-478.

Disclosure of Interest: None declared DOI: 10.1136/annrheumdis-2018-eular.1931

\section{AB0373 SARCOPENIA COULD NOT PREDICT FALLS IN PATIENTS WITH RHEUMATOID ARTHRITIS FROM THE CHIKARA STUDY}

M. Tada ${ }^{1}$, Y. Yamada ${ }^{2}$, K. Mandai ${ }^{2}$, N. Hidaka ${ }^{1} .{ }^{1}$ Orthopaedic Surgery, Osaka City General Hospital; ${ }^{2}$ Orthopaedic Surgery, Osaka City University Graduate School of Medicine, Osaka, Japan

Background: The falls ratio is higher among patients with sarcopenia than among healthy individuals. We reported that the prevalence of sarcopenia was $28 \%$ and locomotive syndrome (locomo) was $52 \%$ in patients with rheumatoid arthritis $(\mathrm{RA})^{1}$. Whether patients with RA complicated by sarcopenia show a higher ratio of falls compared to those without sarcopenia remains unclear.

Objectives: We investigated events of falls and fractures, and predictors of those events in patients with RA

Methods: We used data from a prospective observational study (CHIKARA study, UMIN000023744) started in 2016. Sarcopenia was diagnosed using the criteria of the Asia Working Group on Sarcopenia ${ }^{2}$. We counted the number of patients and events of falls and fractures they had per year[A1], and investigated correlations between those events and disease activity, body composition and sarcopenia. Predictors at baseline influencing those events were analysed by uniand multivariate analysis

Results: Participants comprised 100 patients with RA (females, 78\%; mean age, 66.1 years). Falls occurred in 21 patients (19 women), as 33 events (mean, 2 times/patient). Fractures occurred in 4 patients ( 4 women), as 5 events. Table 1 shows predictors for falls, with positive correlations for bone mass index, obesity level, fat percentage, and locomo, and negative correlations for height, trunk muscle mass, and grip strength. No relationships were seen between falls and CRP, DAS28ESR, skeletal muscle mass, and sarcopenia. Height (odds ratio, $0.912 ; p=0.003$ ) and obesity level (odds ratio, $1.04 ; p=0.006$ ) were independent predictors by multivariate analysis (table 1 ). In terms of fracture events, falls was the only predictor $(r=0.469, p=0.001)$.

Abstract AB0373 - Table 1. Predictors of falls in patients with RA

\begin{tabular}{|c|c|c|c|c|c|}
\hline & \multicolumn{2}{|c|}{ Univariate } & \multicolumn{3}{|c|}{ Multivariate } \\
\hline & $\mathrm{R}$ & $P$ & $\begin{array}{l}\text { Odds } \\
\text { ratio }\end{array}$ & $95 \% \mathrm{Cl}$ & $P$ \\
\hline Body mass index & 0.195 & 0.040 & & & \\
\hline Obesity revel & 0.194 & 0.040 & 1.040 & $\begin{array}{c}1.011- \\
1.069\end{array}$ & 0.006 \\
\hline Fat percentage & 0.211 & 0.025 & & & \\
\hline $\begin{array}{l}\text { Locomotive } \\
\text { syndrome }\end{array}$ & 0.237 & 0.012 & & & \\
\hline Height & -0.263 & 0.005 & 0.912 & $\begin{array}{c}0.858- \\
0.968\end{array}$ & 0.003 \\
\hline Trunk muscle mass & -0.188 & 0.048 & & & \\
\hline Grip strength & -0.198 & 0.036 & & & \\
\hline CRP & 0.063 & 0.512 & & & \\
\hline DAS28ESR & -0.008 & 0.932 & & & \\
\hline $\begin{array}{l}\text { Skeletal muscle } \\
\text { mass }\end{array}$ & 0.091 & 0.337 & & & \\
\hline Sarcopenia & -0.093 & 0.327 & & & \\
\hline
\end{tabular}

Conclusions: Falls have been reported as significantly more frequent with sarcopenia. However, no relationship was seen between fall events and sarcopenia in this study. We may need to evaluate not only skeletal muscle mass, but also muscle function to predict falls.

\section{REFERENCES:}

[1] Tada M, Yamada Y, Mandai K, et al. Relationship of matrix metalloprotease 3 titer and sarcopenia in patients with rheumatoid arthritis: data from the CHIKARA study. Eular 2017:abstract THU0157.

[2] Chen LK, Liu LK, Assantachai P, et al. Sarcopenia in Asia: consensus report of the Asian Working Group for Sarcopenia. J Am Med Dir Assoc 2014;15:95-101.

Disclosure of Interest: None declared DOI: 10.1136/annrheumdis-2018-eular.2562 